\title{
Multiple Input Multiple Output Dirty Paper Coding: System Design and Performance
}

\author{
Zouhair Al-qudah and Dinesh Rajan, Senior Member,IEEE \\ Electrical Engineering Department \\ Southern Methodist University \\ Dallas, Texas 75275 \\ Email: zalqudah, rajand@smu.edu
}

\begin{abstract}
In this paper, a practical multiple input multiple output Dirty paper coding (MIMO-DPC) scheme is designed to cancel the effect of additive interference that is known perfectly to the transmitter. The proposed system uses a trio of encoders - a LDPC code, a vector quantizer implemented as a convolutional decoder and an Orthogonal space time block code (STBC) to achieve temporal coding gain, interference cancelation and spatial diversity, respectively. First, we derive the equivalent noise seen by the receiver using an equivalent lattice based dirty paper code. Then the optimal value of the power inflation factor, which is one of the key system parameters used to minimize the equivalent noise seen by the receiver is derived. Furthermore, we analytically prove that the equivalent noise seen by the receiver tends to 0 for large number of receive antennas. Performance results in the case of various number of receiver antennas are presented and show that significant reduction in bit error probabilities can be obtained over a system that uses no interference cancelation.
\end{abstract}

Index Terms-Dirty Paper Coding, Lattice Coding, Orthogonal Space Time Block Coding .

\section{INTRODUCTION}

Communicating over side information channels, where the interference signal is non-causally available to the transmitter but not to the receiver, has been an active field of research in recent years. Such interference cancelation schemes at the transmitter are commonly known as dirty paper coding (DPC) [1] and are being used in a wide range of applications such as MIMO channels and fading channels [2][4]. The performance of DPC has been widely studied for the single input single output (SISO) channel with perfect noncausal interference [1], faded non-causal interference [5], and noisy causal interference [6]. These capacity limits have been extended to MIMO systems such as the broadcast channel [4] and the cognitive Z-interference channel [7]. Simultaneous to this theoretical developments, practical DPC techniques have also been proposed for the SISO additive Gaussian channel [8], [9], the MIMO broadcast channel [10] and for cognitive radio channels [11].

In this paper, we consider the generalized multiple input multiple output channel with interference as defined in [2], [11]. We develop a practical transceiver structure to leverage knowledge of interference at the transmitter to improve system performance. In more details, we consider an O-STBC system using the DPC to exploit the availability of the non-causal interference only at the transmitter. In order to exploit this interference, the system structure at both the transmitter and the receiver are given. The proposed encoding structure is a combination of a MIMO dirty paper code (DPC), an low density parity check code (LDPC) and a space-time block encoder.

The receiver first employs an space-time combiner followed by an iterative decoder that passes information between the LDPC decoder and the interference mitigating channel decoder. Consider a system with $n_{T}$ transmit antennas and $n_{R}$ receive antennas. Let $\mathbf{X} \in C^{n_{T}}$ be the transmitted signal and let there be a transmit power constraint $\operatorname{Tr}\left(\Sigma_{X}\right) \leq P_{X}$. The multiple input multiple output channel with interference is given by [2], [11]

$$
\mathbf{Y}=\mathbf{H X}+\mathbf{G S}+\mathbf{Z}
$$

where $\mathbf{Y} \in C^{n_{R}}$ is the received signal, $\mathbf{Z} \in C^{n_{R}}$ is the additive white Gaussian noise vector with zero mean and covariance $\Sigma_{Z}$, and $\mathbf{S} \in C^{n_{I}}$ is the interference vector. The number of transmit antennas at the interfering source equals $n_{I}$. This interference is assumed to be non-causally known at the transmitter but not to the receiver. Further $\mathbf{S}$ is assumed to be a zero mean Gaussian distributed random variable with covariance matrix $\Sigma_{S}$ and power $Q=\left|\Sigma_{S}\right|$. The channels $\mathbf{H} \in C^{n_{T} \times n_{R}}$ and $\mathbf{G} \in C^{n_{R}}$ are modeled as quasi static fading matrices from the transmitter and interfering sources, respectively, to the receiver. We assume that these channels are known perfectly to both the transmitter and the receiver [12]. For instance, the receiver could measure both these channels and use a feedback link to send them to the transmitter. The transmitted codeword, the interference and the noise signals are assumed to be independent. Figure 1 shows the general structure for such a system.

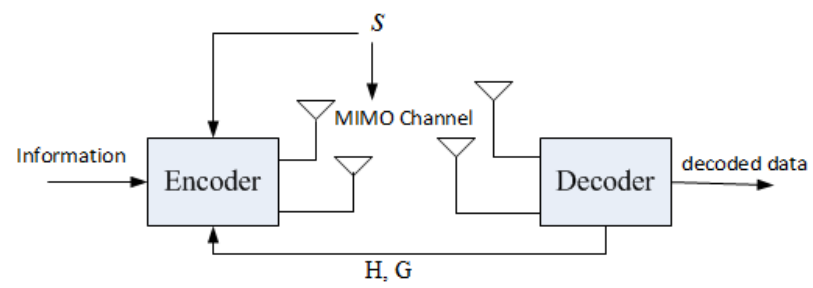

Fig. 1. MIMO Interference channel with interference known at the transmitter. 
The proposed scheme has a scaling parameter which is used to compensate for interference powers that are significantly different from the transmit powers. This scaling factor is typically referred to as the power inflation factor and its choice critically determines the system performance. In this paper, we derive the optimal value of this power scaling factor by considering an equivalent lattice DPC system. In particular, we covert the MIMO-OSTBC channel into its equivalent modulo lattice additive noise (MLAN) channel by using lattice based DPC described in [13]. In more details, we compute at first the equivalent noise seen by the receiver, then the power inflation factor that is used to minimize the equivalent is computed.

For comparison we consider a base line transmission scheme without any DPC that uses a concatenated LDPC and space-time coders similar to those in [14], [15]. In the proposed work, we modify this concatenated structure that also does interference cancelation at the transmitter using a vector quantizer. Our approach is motivated by the simple DPC structures used over SISO Gaussian channels in [8], [9]. In addition to considering a MIMO version of the DPC structure, we study the effect of having knowledge of the channel at the transmitter.

Numerical results indicate that even when the power of the interfering source is comparable to the power of the transmitted signal, the proposed scheme can reduce the effect of the interference by an order of magnitude in some cases. The proposed scheme offers performance within $1 \mathrm{~dB}$ of the performance in an ideal MIMO channel without interference. The variation of the frame error rate for various number of receive antennas is also shown. The effect of increasing the constraint length of the convolutional code that is used for interference cancelation is also studied. For instance, increasing the constraint length from $K=7$ to $K=9$ offers a nearly $0.2 \mathrm{~dB}$ improvement in performance at a frame error rate of 0.01 .

The rest of the paper is organized as follows. In Section II, we introduce the system model, transmitter and receiver structure are fully studied. Lattice DPC which is used to derive both the equivalent noise seen by the receiver and the power inflation factor which is used to minimize the equivalent noise seen by the receiver is studied for MIMO-DPC in section III. System performance and numerical results are presented in section IV. And finally we conclude our paper in section V.

\section{SYSTEM DESCRIPTION}

In this section, we present the basic structure of the proposed encoder and decoder.

\section{A. Transmitter Design}

The proposed structure at the transmitter contains three main blocks: i) an LDPC channel encoder that provide temporal diversity gain, ii) a vector quantizer to compensate for the non-causally known interference, and iii) a space time encoder that provides spatial diversity. Consider the transmission of a $k$ bit information sequence $u=\left(u_{1}, u_{2}, \ldots, u_{k}\right)$. This sequence is first encoded using a rate $1 / 2 \mathrm{LDPC}$ encoder to

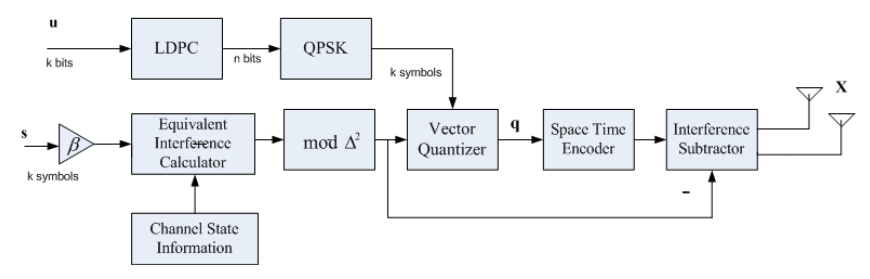

Fig. 2. MIMO-DPC transmit system

generate output $c=\left(c_{1}, c_{2}, \ldots, c_{n}\right)$ of length $n=2 k$. The encoded bits are then mapped to a QPSK constellation from the constellation $\left( \pm \frac{\Delta}{8} \pm j \frac{\Delta}{8}\right)$ to generate $d=\left(d_{1}, d_{2}, \ldots d_{k}\right)$ symbols. Parameter $\Delta$ is selected based on the SNR and interference powers.

The primary objective of the dirty paper coding scheme is to generate a coded sequence that is close to the interference signal. This objective is achieved by generating for each codeword of the LDPC code, a group of equivalent codewords that are distributed over the entire signal space using a procedure that is similar to [8], [9], [16]. Then, the codeword sequence from this group that is closest to the interference signal is selected and will by design have a smaller distance than between the original LDPC codeword and the interfering signal $\tilde{s}$. The group of equivalent symbols for a given symbol $d_{i}$ is formed by shifting the symbol by another QPSK constellation with values $\left( \pm \frac{\Delta}{4} \pm j \frac{\Delta}{4}\right)$ and further considering each value $+k \Delta / 2$ modulo $\Delta$ in both real and imaginary domains. Now, to ensure that the receiver can successfully decode from among these equivalent points, we use a convolutional code to ensure that the selected sequence of symbols is a valid codeword. In effect, these symbols are being processed using a vector quantizer that finds the sequence that is closest to the modified interference signal. The sequence of outputs of the vector quantizer form a valid codeword of the underlying convolutional code. An alternate view is to think of the selected sequence as a valid codeword both for the LDPC code and for the convolutional code.

Finally, a STBC is used to achieve spatial diversity gain over the MIMO channel. For illustration, we focus on the case of using an Orthogonal-STBC (O-STBC). Specifically, the Alamouti code [17] for two transmit antennas is considered.

Each pair of signals, $\left(q_{1} q_{2}\right)$, from the output of the vector quantizer is mapped by the space time encoder to output the symbols over two successive time-instants as:

$$
\left(\begin{array}{ll}
q_{1} & q_{2}
\end{array}\right) \mapsto\left(\begin{array}{cc}
q_{1} & q_{2} \\
-q_{2}^{*} & q_{1}{ }^{*}
\end{array}\right)
$$

where the rows of the matrix represents the temporal and the columns represent the spatial dimension. From the output of the STBC, the modified interference symbols are subtracted before transmission.

In order to calculate the modified interference vector, an interference signal is considered. Furthermore, let $s_{1}$ and $s_{2}$ be the available interference at the transmitter at two consecutive time symbols. These values are scaled by the 
power inflation factor at the transmitter $\beta$ and then fed to a two dimensional modulo operation as depicted in Figure 2. The modified interference vector is then subtracted from the quantized symbols at the output of the vector quantizer to generate the symbols to be transmitted as,

$$
\left(\begin{array}{cc}
q_{1}-s_{11}^{\prime} & q_{2}-s_{22}^{\prime} \\
-q_{2}{ }^{*}+s_{22}^{*^{\prime}} & q_{1}^{*}-s_{11}^{*^{\prime}}
\end{array}\right)
$$

where

$$
s_{i j}^{\prime}=\beta s_{i j} \bmod \Delta^{2}, \quad i, j=1,2
$$

represents the modified interference symbol that will be compensated by antenna $j$ at time-instant $i, s_{i j}^{\prime}$ will be computed later.

The optimal value of the power inflation factor $\beta$ is derived using Lattice based codes approach similar to [5], [8], [13], [16], [18].

\section{B. Iterative Receiver Design}

The received signal, $r_{t l}$ at time $t \in\{1,2\}$ and antenna $l$ is given by,

$$
\begin{aligned}
& r_{11}=h_{11}\left(q_{1}-s_{11}^{\prime}\right)+h_{21}\left(q_{2}-s_{22}^{\prime}\right)+s_{1}+n_{1} \\
& r_{12}=h_{12}\left(q_{1}-s_{11}^{\prime}\right)+h_{22}\left(q_{2}-s_{22}^{\prime}\right)+s_{1}+n_{2} \\
& r_{21}=h_{11}\left(-q_{2}{ }^{*}+s_{22}^{*^{\prime}}\right)+h_{21}\left(q_{1}{ }^{*}-s_{11}^{*^{\prime}}\right)+s_{2}+n_{3} \\
& r_{22}=h_{12}\left(-q_{2}{ }^{*}+s_{22}^{*^{\prime}}\right)+h_{22}\left(q_{1}{ }^{*}-s_{11}^{*^{\prime}}\right)+s_{2}+n_{4}
\end{aligned}
$$

where $h_{t j}=\alpha_{t j} e^{i \theta_{t j}}$ represents the channel gain from transmit antenna $t$ to receive antenna $j$.

The receiver structure is shown in Figure 3. The received signals are combined using knowledge of the channel fading $\mathbf{H}$ to generate an estimate of the signals $q_{1}$ and $q_{2}$ as

$$
\begin{aligned}
\tilde{q_{1}}= & h_{11}^{*} r_{11}+h_{12}^{*} r_{12}+h_{21} r_{21}{ }^{*}+h_{22} r_{22}{ }^{*} \\
= & \alpha_{11}^{2}\left(q_{1}-s_{11}^{\prime}\right)+h_{11}^{*} s_{1}+h_{11}^{*} n_{1} \\
& +\alpha_{12}^{2}\left(q_{1}-s_{11}^{\prime}\right)+h_{12}^{*} s_{1}+h_{12}^{*} n_{2} \\
& +\alpha_{21}^{2}\left(q_{1}-s_{11}^{\prime}\right)+h_{21} s_{2}{ }^{*}+h_{21} n_{3}{ }^{*} \\
& +\alpha_{22}^{2}\left(q_{1}-s_{11}^{\prime}\right)+h_{22} s_{2}{ }^{*}+h_{22} n_{4}{ }^{*}
\end{aligned}
$$

$$
\begin{aligned}
\tilde{q_{2}}= & h_{21}^{*} r_{11}+h_{22}^{*} r_{12}-h_{11} r_{21}{ }^{*}-h_{12} r_{22}{ }^{*} \\
= & \alpha_{21}^{2}\left(q_{2}-s_{22}^{\prime}\right)+h_{21}^{*} s_{1}+h_{21}^{*} n_{1} \\
& +\alpha_{22}^{2}\left(q_{2}-s_{22}^{\prime}\right)+h_{22}^{*} s_{1}+h_{22}^{*} n_{2} \\
& +\alpha_{11}^{2}\left(q_{2}-s_{22}^{\prime}\right)-h_{11} s_{2}{ }^{*}-h_{11} n_{3}{ }^{*} \\
& +\alpha_{12}^{2}\left(q_{2}-s_{22}^{\prime}\right)-h_{12} s_{2}{ }^{*}-h_{12} n_{4}{ }^{*}
\end{aligned}
$$

We now derive the values of $s_{11}$ and $s_{22}$ to ensure that the combined signals at the receiver are free of interference. From (9) and (10), the following are obtained

$$
\begin{aligned}
& s_{11}=\left(\left(h_{11}^{*}+h_{12}^{*}\right) s_{1}+\left(h_{21}+h_{22}\right) s_{2}^{*}\right) / \alpha_{2} \\
& s_{22}=\left(\left(h_{21}^{*}+h_{22}^{*}\right) s_{1}-\left(h_{11}+h_{12}\right) s_{2}^{*}\right) / \alpha_{2}
\end{aligned}
$$

where $\alpha_{2}=\left(\alpha_{11}^{2}+\alpha_{12}^{2}+\alpha_{21}^{2}+\alpha_{22}^{2}\right)$. Substituting these values in (3), the effective signal in (9) is reduced to

$$
\begin{array}{r}
\tilde{q_{1}=\alpha_{11}^{2}}\left(q_{1}-s_{11}^{\prime}\right)+\alpha_{12}^{2}\left(q_{1}-s_{11}^{\prime}\right)+\alpha_{21}^{2}\left(q_{1}-s_{11}^{\prime}\right) \\
+\alpha_{22}^{2}\left(q_{1}-s_{11}^{\prime}\right)+\alpha_{11}^{2} s_{11}+\alpha_{12}^{2} s_{11}+\alpha_{21}^{2} s_{11} \\
+\alpha_{22}^{2} s_{11}+h_{11}^{*} n_{1}+h_{12}^{*} n_{2}+h_{21} n_{3}^{*}+h_{22} n_{4}^{*}
\end{array}
$$

The combined signals are then multiplied by the power inflation factor $\beta$ and then processed by a non-linear modulo operation $\bmod \Delta^{2}$ to ensure that interference free received signals are obtained. These operations will be more clear in section III. Finally these signals are fed to an iterative decoder to obtain the transmitted data sequence $u$. In the interest of space, we only provide a brief description of the iterative decoder; see [8], [15], [19] for additional details. As noted before, the signal $q$ is a codeword both of the convolutional code as well as the LDPC code. The iterative decoding works as follows. First we use the BCJR type MAP decoder for the convolutional code (BCJR-VQ) [8], [9] that computes the a-posteriori information of the symbol sequence $q_{i}^{\prime}$ where $i \in(1, \ldots, k)$ based on the received signal and the a-priori information provided by the channel.

Then, the soft de-mapper computes the extrinsic information that are passed to the LDPC decoder as [15], [20]

$$
L L R_{12}\left(c_{i}^{j}\right)=\ln \frac{\sum_{c_{i}^{j}=1} e^{L L R_{0}\left(c_{i}\right)}}{\sum_{c_{i}^{j}=0} e^{L L R_{0}\left(c_{i}\right)}}
$$

where $j \in\{1,2\}$ represents the bit position in symbol $i$.

The LDPC decoder employs the sum product algorithm (SPA) which gives a-posteriori probabilities about the LDPC coded bits. In the next iteration, this a-posterior information is passed as the new a-priori information to the BCJRVQ decoder. The iteration stops after the desired stopping criterion is met, and the LDPC decoder outputs the decoded codeword and the a-posteriori probabilities of the data bits is computed [15], [20] by the soft-mapper as

$$
L L R_{21}\left(c_{i}=c_{i}^{1} c_{i}^{2}\right)=\prod_{j=1}^{2} \frac{e^{c_{i}^{j} L L R_{2}\left(c_{i}^{j}\right)}}{1+e^{L L R_{2}\left(c_{i}^{j}\right)}}
$$

where $L L R_{2}=L L R_{1}-L L R_{12}, L L R_{1}$ is extrinsic information provided by the SPA algorithm. Essentially, the soft mapper and de-mapper relate the bit a-priori probabilities with the symbol a-priori probabilities.

Numerical results that indicate the performance of the proposed scheme are presented in Section IV. Next, we discuss a method that is used to determine the optimal value of the power inflation factors $\beta_{i}$. 


\section{LATtice DirTy PAPER CODING FOR MIMO-STBC}

We convert the MIMO-OSTBC into its equivalent modulo lattice additive noise (MLAN) channel. This MLAN channel contains only the transmitted quanitized vector $q$ corrupted by equivalent additive noise seen by the receiver. The equivalent noise seen by the receiver is computed using lattice based DPC. After that, we compute the power inflation factors $\beta_{i}$ which is used to both the transmitter and receiver to minimize the effective noise power seen at the receiver. In our derivation, we follow the approach described in [8], [13] .

We first derive the equivalent noise seen by the receiver and then the power inflation factor for a $2 \times 2$ MIMO-STBC in Section III-A. Generalizations to the case of arbitrary number of receive antenna is given in Section III-B.

\section{A. Equivalent Noise and Power Inflation Factor for $2 \times 2$ MIMO-STBC}

Consider the transmission of a data sequence of length $n$ bits. Group these bits into $l$ blocks where each block contains $n / l$ bits. The entire received signal $y$ contains $n / l$ elements, i.e., $y=\left(y_{1}, \ldots, y_{n / l}\right)$. In block $i$, the receiver computes $y_{i}{ }^{\prime}=\left[\beta_{i} q_{i}\right] \bmod \Lambda$, where $\beta_{i}$ is the power inflation factor at the transmitter. To model the proposed system using (12), the estimated value of $\tilde{q}_{i}$ is first multiplied by the power inflation factor and then fed to a non-linear modulo operation. Let $x=q_{1}-s_{11}^{\prime}$, so that (12) can be re-written as

$$
\begin{aligned}
y_{i}{ }^{\prime}= & {\left[\beta_{i} \alpha_{2} x+\beta_{i} \alpha_{2} s_{11}+\beta_{i} h_{11}^{*} n_{1}\right.} \\
& \left.+\beta_{i} h_{12}^{*} n_{2}+\beta_{i} h_{21} n_{3}^{*}+\beta_{i} h_{22} n_{4}^{*}\right] \bmod \Lambda
\end{aligned}
$$

After substituting the values of $s_{11}^{\prime}$ and then doing some simple algebraic manipulations, (15) can be re-written as

$$
\begin{gathered}
y_{i}{ }^{\prime}=\left[\alpha_{2} q_{1}-\alpha_{2} x\left(1-\beta_{i}\right)+h_{11}^{*} \beta_{i} n_{1}\right. \\
\left.+h_{12}^{*} \beta_{i} n_{2}+h_{21} \beta_{i} n_{3}{ }^{*}+h_{22} \beta_{i} n_{4}{ }^{*}\right] \bmod \Lambda
\end{gathered}
$$

In this derivation, we used the modulo-lattice distributive property [21]

$$
[(x \bmod \Lambda)+y] \bmod \Lambda=[x+y] \bmod \Lambda
$$

The receiver now attempts to get the transmitted codeword by decoding the sequence $y^{\prime}=\left(y_{1}^{\prime}, \ldots, y_{n / l}{ }^{\prime}\right)$. Equation (16) contains the transmitted codeword corrupted by additive noise. The equivalent noise $z_{i}{ }^{\prime}$ at the receiver is given by,

$$
\begin{aligned}
z_{i}{ }^{\prime} & =\frac{1}{\alpha_{2}}\left[-\alpha_{2} x\left(1-\beta_{i}\right)+h_{11}^{*} \beta_{i} n_{1}\right. \\
& \left.+h_{12}^{*} \beta_{i} n_{2}+h_{21} \beta_{i} n_{3}{ }^{*}+h_{22} \beta_{i} n_{4}{ }^{*}\right] \bmod \Lambda
\end{aligned}
$$

Equation (18) contains noise due to quantization at both the transmitter and the receiver and AWGN. The transmitted symbols $x_{i}$ are assumed to be independent and have equal power $P_{x}$ on average, the noise corrupting the receiver at different times is also assumed to be independent and have equal power $P_{n}$. The variance of the effective noise is computed as

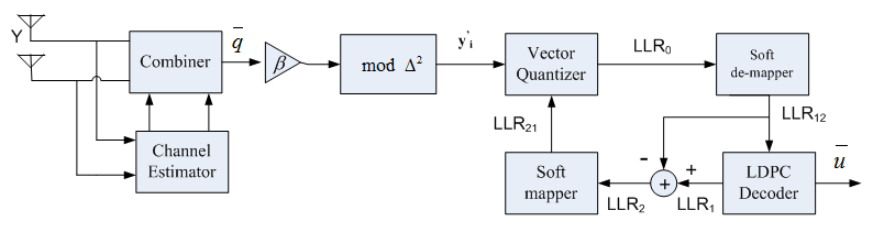

Fig. 3. MIMO-DPC receiver system

$$
P_{z_{i}{ }^{\prime}}=\frac{1}{\alpha_{2}^{2}}\left[\alpha_{2}^{2}\left(1-\beta_{i}\right)^{2} P_{x}+\alpha_{2} \beta_{i}^{2} P_{n}\right]
$$

We now minimize this equivalent noise power given in (19) over all values of $\beta_{i}$. For fixed values of $P_{x}$ and $P_{n}$, we set the first derivative of $P_{z_{i}{ }^{\prime}}$ with respect to $\beta_{i}$ equal to 0 to obtain the power inflation factor as

$$
\beta_{i}=\frac{\alpha_{2} P_{x}}{\alpha_{2} P_{x}+P_{n}}
$$

With this choice of $\beta_{i}$, the equivalent noise in (19) reduces to

$$
P_{z_{i}{ }^{\prime}}=\frac{P_{x} P_{n}}{\alpha_{2} P_{x}+P_{n}}
$$

Finally, we note that the formula in (16) can be re-written as

$$
y_{i}{ }^{\prime}=\left[q_{i}+z_{i}{ }^{\prime}\right] \bmod \Lambda
$$

which is effectively the transmitted signal corrupted by additive noise with variance $P_{z_{i}{ }^{\prime}}$. Clearly, the MIMO channel described in this paper is fully described by the modulo channel. We refer the reader to [18] for more details on the modulo channel.

Finally we note that the power inflation factor formula given in (20) is valid for arbitrary values of both the interference power and the number of antennas at the interference source since it does not depend on both.

\section{B. Power Inflation Factor for $2 \times n_{R}$ MIMO-STBC}

In this subsection, the equivalent noise seen by the receiver and the associated power inflation factor are generalized to the case of two transmit antennas and an arbitrary number of receive antennas. We start by noting that

$$
\alpha_{n_{R}}=\left(\alpha_{11}^{2}+\ldots+\alpha_{1 n_{R}}^{2}+\alpha_{21}^{2}+\ldots+\alpha_{2 n_{R}}^{2}\right)
$$

For this setting, the equivalent noise seen by the receiver $P_{z_{i}^{\prime}}$ can be easily shown to be

$$
P_{z_{i}^{\prime}}=\frac{1}{\alpha_{n_{R}}^{2}}\left(\alpha_{n_{R}}^{2}\left(1-\beta_{i}\right)^{2} P_{x}+\alpha_{n_{R}} \beta_{i}^{2} P_{n}\right)
$$

Then the associated power inflation factor for this setting can be easily derived by setting the derivative of $P_{z_{i}^{\prime}}$ in (24) respect to $\beta_{i}$ equal to 0 to obtain the power inflation factor in (25)

$$
\beta_{i}=\frac{\alpha_{n_{R}} P_{x}}{\alpha_{n_{R}} P_{x}+P_{n}}
$$


Again,with this choice of $\beta_{i}$, the equivalent noise in (24) reduces to

$$
P_{z_{i}}=\frac{P_{x} P_{n}}{\alpha_{n_{R}} P_{x}+P_{n}}
$$

\section{Results AND Discussion}

We now present numerical results of the proposed system. The transmitter uses a rate $1 / 2$ LDPC with a generator matrix of size $(252,504)$ having 3 ones per row [22] and frame size of 252 symbols. A rate $1 / 2$ convolutional encoder which is employed as a vector quantizer by means of Viterbi algorithm at the transmitter and BCJR at the receiver with constraint length $K=7$ and generator polynomials $(171,133)$ is used. These values of the generator polynomials give the best performance for the given constraint length [23]. Moreover, our results have been obtained for 3 turbo decoding iterations and 150 iterations for the LDPC-decoder. For a given pair of transmit and receive antennas $\left(n_{T}=2, n_{R}\right)$ the power inflation factor which is used to minimize the equivalent noise seen by the receiver is first computed using the derived formulas and then used in our simulations. As noted before, the Alamouti scheme is used as the space-time code to transmit the signal. In our results we set the power of the interference as equal to the power of the transmitted signal. Finally, we note that the complexity of the iterative decoding techniques was extensively studied in [24].

Figure 4 shows the frame error rate (FER) for three systems. First, for comparison we show the FER for a MIMO system without interference. Second, we show the performance of a system without any interference cancelation. These two results act as the lower bound and upper bound on the FER of any system that employs dirty paper coding. Third, we show the performance of the proposed MIMO-DPC system. The results are shown for various number of receive antennas. Since the interference power is equal to the signal transmit power, the FER of a system without interference cancellation does not reduce with increasing SNR. Clearly, the FER of the proposed MIMO-DPC system reduces as SNR increases. For instance, in a $2 \times 2$ system at a FER of $1 \%$, the proposed scheme is approximately $1.0 \mathrm{~dB}$ away from the performance of the interference free system. Particulary, in Appendix-B, we analytically prove that the equivalent noise power tends to 0 as the number of receive antennas becomes very large.

Figure 5 shows how the performance of the proposed MIMO-DPC scheme can be improved when the constraint length of the convolutional encoder is increased. Specifically, a convolutional encoder with constraint length $K=9$ and generator polynomials $(561,753)$ [23] is used. The reduction in FER is evident for various receive antenna configurations. In this case, the FER of a $2 \times 2$ system is only $1 \mathrm{~dB}$ away from the interference free system. This performance gap can be further reduced by using a convolutional code with higher constraint length but at the cost of increased processing complexity at both transmitter and receiver.

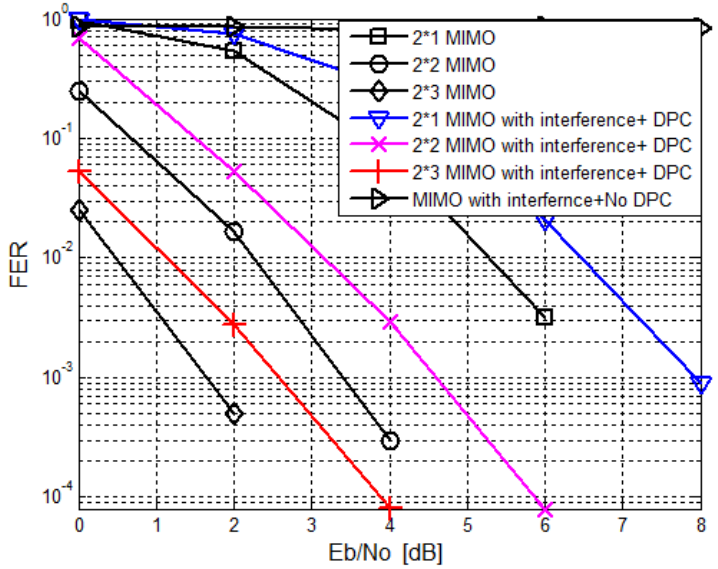

Fig. 4. Comparison between MIMO system without interference and MIMO system with interference which uses DPC to cancel the interference's effect

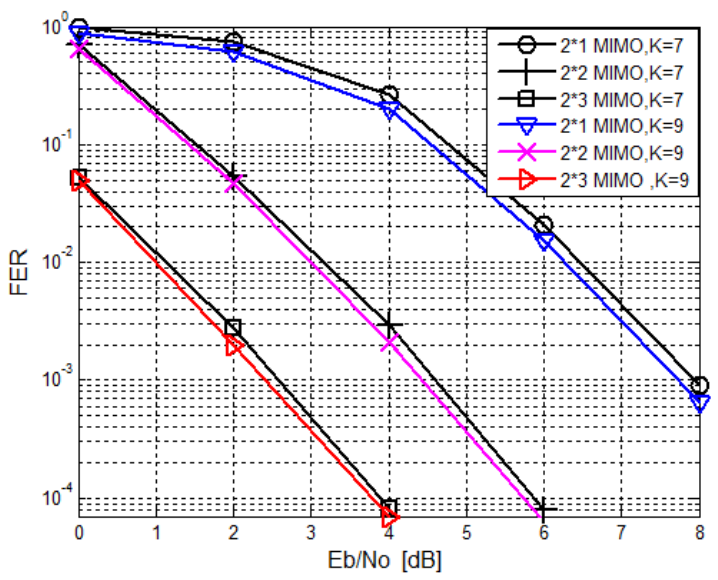

Fig. 5. Performance of MIMO-DPC over different constraint length of the convolutional encoder

\section{CONCLusions}

A MIMO-DPC based on STBC is proposed and its performance is shown for various number of receive antennas. Both the equivalent noise seen by the receiver and the optimal power inflation factor for such a system are derived. Future work should consider the case of errors and causal knowledge of interference at the transmitter as well as non-orthogonal space-time codes.

\section{APPENDIX A ASYMPTOTIC RESULT}

In this appendix, we evaluate the equivalent noise seen by the receiver $P_{z_{i}^{\prime}}$ asymptotically as the number of receive antennas becomes large. We prove that as the number of the receive antenna $n_{R}$ tends to $\infty$, the equivalent noise seen by the receiver tends to 0 . We start by noting that

$$
\alpha_{\infty}=\left(\alpha_{11}^{2}+\alpha_{12}^{2}+\ldots \alpha_{1 \infty}^{2}+\alpha_{21}^{2}+\alpha_{22}^{2}+\ldots+\alpha_{2 \infty}^{2}\right)
$$


We now evaluate the power inflation factor in (25) as the number of receive antennas becomes large $\left(n_{R} \longrightarrow \infty\right)$,

$$
\begin{aligned}
\lim _{n_{R} \longrightarrow \infty} \beta_{i} & =\frac{\alpha_{\infty} P_{x}}{\alpha_{\infty} P_{x}+P_{n}} \\
& =\frac{P_{x}}{P_{x}+\frac{1}{\alpha_{\infty}} P_{n}} \\
& \cong 1 .
\end{aligned}
$$

Now, the equivalent noise power given in (24) is evaluated by substituting the value of $\beta_{i}$ obtained in (28). Clearly, the first term in (24) vanishes as $\beta_{i} \longrightarrow 1$ and in the limit $n_{R} \longrightarrow \infty$,

$$
\begin{aligned}
\lim _{n_{R} \longrightarrow \infty} P_{z_{i}^{\prime}} & =\lim _{n_{R} \longrightarrow \infty} \frac{1}{\alpha_{\infty}} P_{n} \\
& \cong 0
\end{aligned}
$$

This is a parallel result to that obtained in [25] where the error probability tends to 0 for two transmit antenna and large number of receive antennas.

\section{REFERENCES}

[1] M. Costa, "Writing on dirty paper (corresp.)," IEEE Trans. on Info. Theory, vol. 29, pp. 439 - 441, May 1983.

[2] C. Vaze and M. Varanasi, "Dirty paper coding for the MIMO cognitive radio channel with imperfect CSIT," in IEEE Inter. Symp. on Info. Theory, pp. 2532 -2536, 282009-july3 2009.

[3] C. Vaze and M. Varanasi, "Dirty paper coding for fading channels with partial transmitter side information," in 42nd Asilomar Conf. on Signals, Systems and Computers, pp. 341 -345, 2008.

[4] A. Bennatan and D. Burshtein, "On the fading-paper achievable region of the fading MIMO broadcast channel," IEEE Trans. on Info. Theory, vol. 54, no. 1, pp. $100-115,2008$.

[5] A. Bennatan, V. Aggarwal, Y. Wu, A. Calderbank, J. Hoydis, and A. Chindapol, "Bounds and lattice-based transmission strategies for the phase-faded dirty-paper channel," IEEE Trans. on Wireless Comm., vol. 8, no. 7, pp. $3620-3627,2009$.

[6] Y. Peng and D. Rajan, "Achievable rate of gaussian cognitive Zinterference channel with partial side information," in in Proc. of IEEE -GlobeCom, Dec. 2009.

[7] Y. Peng and D. Rajan, "Capacity bounds for cognitive MIMO gaussian Z-interference channel," IEEE Trans. on Vehicular Techn., vol. 59, pp. 1865 - 1876, 2010.

[8] M. Mazzotti and M. Chiani, "A simple rate-1/2 co-decoding scheme for writing on dirty paper," in IEEE Inter. Conf. on Comm. ( ICC '06), pp. $1622-1627,2006$.

[9] G. B. Kyung and C.-C. Wang, "On the designs and challenges of practical binary dirty paper coding," in IEEE -WCNC., 2009.

[10] S.-C. Lin and H.-J. Su, "Practical vector dirty paper coding for MIMO gaussian broadcast channels," IEEE Journal on Selected Areas in Communications, vol. 25, pp. 1345 -1357, september 2007.

[11] P.-H. Lin, S.-C. Lin, C.-P. Lee, and H.-J. Su, "Cognitive radio with partial channel state information at the transmitter," IEEE Trans. on Wireless Comm., vol. 9, no. 11, pp. 3402 -3413, 2010.

[12] A. Jovicic and P. Viswanath, "Cognitive radio: An information-theoretic perspective," IEEE Transactions on Information Theory, vol. 55, pp. 3945 -3958, sept. 2009.

[13] U. Erez, S. Shamai, and R. Zamir, "Capacity and lattice strategies for cancelling known interference," IEEE Trans. on Info. Theory, vol. 51, pp. 3820-3833, 2005.

[14] J. Zhang and H.-N. Lee, "Performance analysis on LDPC-coded systems over quasi-static (MIMO) fading channels," IEEE Trans. on Comm. vol. 56, no. 12 , pp. $2080-2093,2008$.

[15] E. Aydin and I. Altunbas, "Serially concatenated LDPC and spacetime trellis codes with transmit antenna selection," in 14th IEEE Signal Process. and Comm. Applications, pp. 1 -4, 2006.

[16] U. Erez and S. Brink, "A close-to-capacity dirty paper coding scheme," IEEE Trans. on Info. Theory, vol. 51, no. 10, pp. 3417 - 3432, 2005.
[17] S. Alamouti, "A simple transmit diversity technique for wireless communications," IEEE J. on Sel. Areas in Comm., vol. 16, pp. 1451 -1458, Oct. 1998.

[18] R. F. Fischer, "The modulo-lattice channel: The key feature in precoding schemes," International Journal of Electronics and Communications, pp. 244-253, Oct. 2005.

[19] W. Ryan, Concatenated convlutional codes and iterative decoding, p. Wiley Encyclopedia of Telecom. John Wiley \& Sons, 2003.

[20] B.Vucetic and J.Yuan, Space -Time Coding. John Wiley \& Sons, 2003.

[21] Richard.Blahut, Theory and Practice of Error Control Codes. AddisonWesley, 1983.

[22] J. Chen and M. Fossorier, "Near optimum universal belief propagation based decoding of low-density parity check codes," IEEE Trans. on Comm., vol. 50, pp. $406-414$, Mar. 2002.

[23] K. Z. Rolf Johannesson, Fundamentals of Convlutional Coding. John Wiley and Sons, 1999.

[24] G. B. Kyung and C.-C. Wang, "Toward a practical scheme for binary broadcast channels with varying channel quality using dirty paper coding," IEEE Transactions on Communications, vol. 59, pp. 1009 1018, april 2011.

[25] E. Biglieri, G. Taricco, and A. Tulino, "Performance of Space-time Codes for a Large Number of Antennas," IEEE Transactions on Information Theory, vol. 48, pp. 1794 -1803, jul 2002. 